\title{
Electron Orderings of Half-Filled Extended Hubbard Models with Spin- and Charge-Exchange Interaction
}

\author{
W.R. Czart and S. Robaszkiewicz \\ Institute of Physics, A. Mickiewicz University \\ Umultowska 85, 61-614 Poznań, Poland
}

The electron orderings of the half-filled extended Hubbard models (i) with anisotropic spin-exchange interactions (the $t-U-J_{\|}-J_{\perp}$ model) and (ii) with charge-exchange interaction (the $t-U-I$ model) are discussed. In particular, we present new results concerning the phase diagrams of the model (i) in the case of uniaxial spin exchange $\left(J_{\|} \neq 0\right)$ for $d$-dimensional hypercubic lattices $(1 \leq d \leq \infty)$ and conclude about the basic effects of the particular spin- and charge-exchange interaction terms $\left(J_{\|}, J_{\perp}\right.$, and $\left.I\right)$ in both models.

PACS numbers: 71.10.Fd, 75.10.-b, 75.30.Gw, 71.45.Lr, 74.20.--z

\section{Introduction}

The extended Hubbard models (EHM) (i) with anisotropic spin-exchange interaction (the $t-U-J_{\|}-J_{\perp}$ model [1-5]) and (ii) with charge-exchange (pair hopping) interaction (the $t-U-I$ model, which is also called the Penson-KolbHubbard model [6-10]) are interesting (conceptually simple) phenomenological models for studying correlations and for description of superconductivity, magnetism, and various other types of electron orderings in narrow band systems.

The model Hamiltonians have the following form:

(i) the $t-U-J_{\|}-J_{\perp}$ model:

$$
H=H_{U}+\frac{1}{2} \sum_{\langle i, j\rangle} J_{\perp}\left(\sigma_{i}^{\dagger} \sigma_{j}^{-}+\text {h.c. }\right)+\sum_{\langle i, j\rangle} J_{\|} \sigma_{i}^{z} \sigma_{j}^{z},
$$

(ii) the $t-U-I$ model:

$$
H=H_{U}-I \sum_{\langle i, j\rangle} c_{i \uparrow}^{\dagger} c_{i \downarrow}^{\dagger} c_{j \downarrow} c_{j \uparrow},
$$

where

$$
H_{U}=\sum_{\langle i, j\rangle \sigma} t_{i j} c_{i \sigma}^{\dagger} c_{j \sigma}+U \sum_{i} n_{i \uparrow} n_{i \downarrow},
$$


$t$ is the single electron hopping integral, $U$ is the on-site density interaction, $J_{\perp}$ and $J_{\|}$are $X Y$ and $Z$ components of intersite magnetic exchange interaction, respectively, $I$ is the charge-exchange interaction. The spin operators $\left\{\boldsymbol{\sigma}_{i}\right\}$ are defined by $\sigma_{i}^{z}=\frac{1}{2}\left(n_{i \uparrow}-n_{i \downarrow}\right), \sigma_{i}^{+}=c_{i \uparrow}^{+} c_{i \downarrow}=\left(\sigma_{i}^{-}\right)^{+}$.

In our recent works we have performed extensive analysis of the phase diagrams and thermodynamic and electromagnetic properties of the model (2) both for attractive $(I>0)$ and repulsive $(I<0)$ charge-exchange interaction, for various lattice structures and arbitrary electron concentration $0<n<2$ [6-9]. We have also studied the properties of the model (1), in the case of transverse (XY-type) spin exchange of either $\operatorname{sign}\left(J_{\perp} \neq 0, J_{\|}=0\right)[4,9]$. In the analysis we have used the (broken symmetry) Hartree-Fock approximation (HFA) supplemented, for $d=\infty$, by the slave boson mean-field approach (SBMFA) $[6,8]$ and for $d=1$ by the density-matrix renormalization group (DMRG) method [9]. Additionally, the variational approach, which treats the on-site interaction term exactly, has been applied in the analysis of the $t=0$ case [4].

In this work we extend these studies presenting new results concerning the phase diagrams of the model (1) at half-filling in the case of uniaxial spin exchange $J_{\|} \neq 0$ and conclude about the basic effects of the particular spin- and charge-exchange interaction terms $\left(J_{\perp}, J_{\|}\right.$, and $\left.I\right)$ for both models.

\section{Results and discussion}

The ground state diagrams of the half-filled $t-U-J_{\|}$model determined within (broken symmetry) HFA are shown in Fig. 1 for the $d=2$ SQ lattice (Fig. 1a) and for the $d=\infty$ hypercubic lattice (Fig. 1b). The SBMFA phase diagram for $d=\infty$ is almost identical to that in Fig. 1b, although the values of the
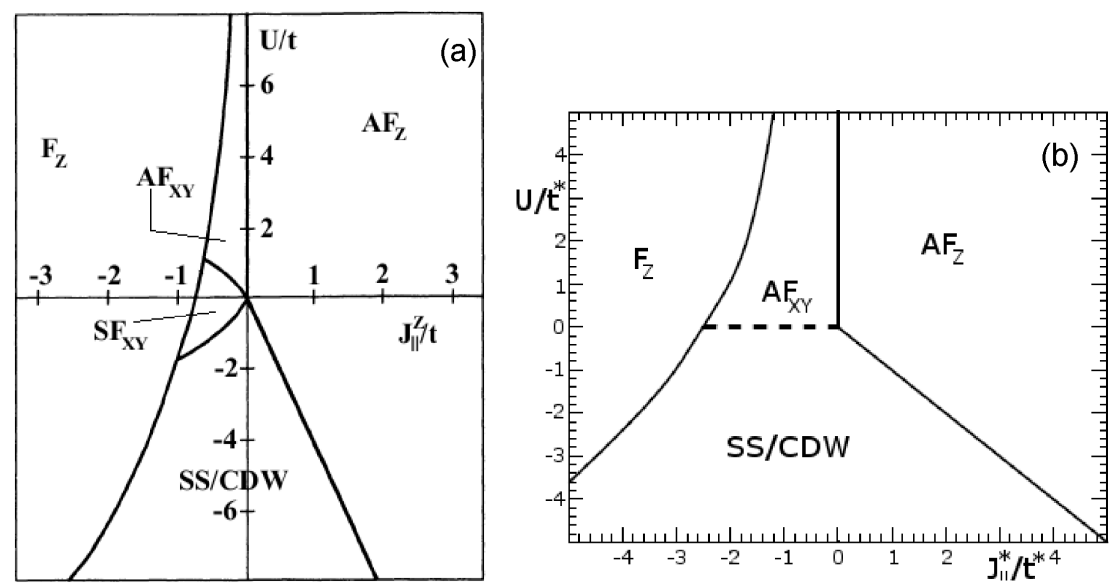

Fig. 1. Ground state phase diagrams of the half-filled $t-U-J_{\|}$model (a) for the $d=2$ SQ lattice and (b) for the $d=\infty$ hypercubic lattice determined within the (broken symmetry) HFA. The SBMFA phase diagram for $d=\infty$ is almost identical to (b). In (b): $t^{*}=t \sqrt{d}, J_{\|}^{*}=J_{\|}$. 
order parameters and the energy gaps in all the phases are substantially reduced by the correlation effects.

For $d=1$, going beyond the mean field approaches we have used the level crossing approach for finite-size clusters to determine the phase boundaries, analogous to that applied previously for the EHM with intersite Coulomb interaction by Nakamura [11]. In this treatment the transition points are identified by the level crossing of the excitation spectra in the finite-size ring with size dependence $O\left(L^{-2}\right)$, where $L$ is the system size. The resulting diagram for $L=12$ is presented in Fig. 2.

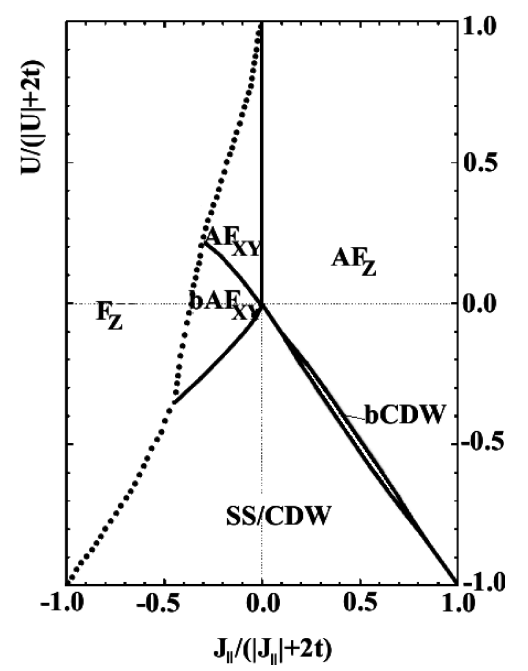

Fig. 2. Ground state phase diagram of the half-filled $t-U-J_{\|}$model for the $1 \mathrm{D}$ chain, obtained numerically, using level-crossing approach for finite-size clusters $(L=12)$.

For $d=\infty$ the phase diagram involves exclusively the site-located orderings: uniaxial ferromagnetic $\left(\mathrm{F}_{Z}\right)$, with $x_{\mathrm{F}_{Z}}=\frac{1}{N} \sum_{i}\left\langle\sigma_{i}^{Z}\right\rangle \neq 0$, and antiferromagnetic $\left(\mathrm{AF}_{Z}\right)$, with $x_{\mathrm{AF}_{Z}}=\frac{1}{N} \sum_{i}\left\langle\sigma_{i}^{Z}\right\rangle \exp \left(\mathrm{i} \boldsymbol{Q} \boldsymbol{R}_{i}\right) \neq 0$, phases, the planar antiferromagnetic $\left(\mathrm{AF}_{X Y}\right)$ phase, with $x_{\mathrm{AF}_{X Y}}=\frac{1}{N} \sum_{i}\left\langle\sigma_{i}^{+}\right\rangle \exp \left(\mathrm{i} \boldsymbol{Q} \boldsymbol{R}_{i}\right) \neq 0$, as well as the $s$-wave superconducting (SS) and CDW phases, which for $n=1$ are strictly degenerated, and therefore the corresponding state is denoted by SS/CDW (Fig. 1).

For $d \leq 3$ the model can also exhibit various bond-located orderings. They are realized for $J_{\|}<0$ in the weak/intermediate coupling regime: the bond $\mathrm{AF}_{X Y}$ order $\left(\mathrm{bAF}_{X Y}\right)$ for $d=1$ and the spin flux (spin nematic) order $\left(\mathrm{SF}_{X Y}\right)$ for $d=2$, as well as for $J_{\|}>0$, close to the boundary between $\mathrm{AF}_{Z}$ and SS/CDW phases: the bond CDW order (bCDW) for $d=1$ (cf. Fig. 1a and Fig. 2).

The phase diagram for $d=1$ derived within HFA [9] is similar to that shown in Fig. 2 except that close to the boundary lines separating the $\mathrm{AF}_{X Y}$ and $\mathrm{bAF}_{X Y}$ states as well as the $\mathrm{bAF}_{X Y}$ and $\mathrm{SS} / \mathrm{CDW}$ states there are found narrow regions 
of the mixed ordered phases $\left(\mathrm{AF}_{X Y}+\mathrm{bAF}_{X Y}\right.$ for $U>0$ and $\mathrm{SS} / \mathrm{CDW}+\mathrm{bAF}{ }_{X Y}$, for $U<0)$.

In any dimension, the transition at $T=0$ to $\mathrm{F}_{Z}$ phase (which is favored by $\left.J_{\|}<0\right)$ is of the first order and occurs only above some critical value of $\left|J_{\|}\right| / D$. This is in obvious contrast with the properties of $\mathrm{AF}_{Z}$ phase, which at $U \geq 0$ exhibits a smooth crossover from the weak coupling limit to the local magnetic moment regime with increasing $J_{\|}>0$. Repulsive $U$ expands the range of stability of $\mathrm{F}_{Z}$ phase at $T=0$ towards lower values of $\left|J_{\|}\right|$. Moreover the $\mathrm{F}_{Z}$ and $\mathrm{AF}_{Z}$ phases can survive also for attractive values of $U\left(0>U>U_{c}\right.$, cf. Figs. 1, 2).

We have also studied the phase diagrams of the model (1) in the case of isotropic spin exchange: $J_{\perp}=J_{\|}=J$, for which the system has $S U(2)$ spin symmetry. For $d=\infty$ the phase diagrams determined within the HFA, as well as in SBMFA are identical to that given in Fig. 1b, if one replaces the denotations: $J_{\|} \rightarrow J, \mathrm{~F}_{X Y} \Rightarrow \mathrm{F}$ and $\mathrm{AF}_{X Y}, \mathrm{AF}_{Z} \Rightarrow \mathrm{AF}[9]$. On the other hand, the form of the diagrams in the isotropic case for $d \leq 3$ can be essentially different than that of those for the uniaxial exchange. For example, in Fig. 3 we show the phase diagram of the $t-U-J$ model obtained within HFA for $d=2 \mathrm{SQ}$ lattice. Let us notice the existence of the phases with $d$-wave superconducting order (DS), absent in the half-filled $t-U-J_{\|}$model and appearing close to the boundary between SS/CDW and AF phases (comp. Fig. 3 with Fig. 1a).

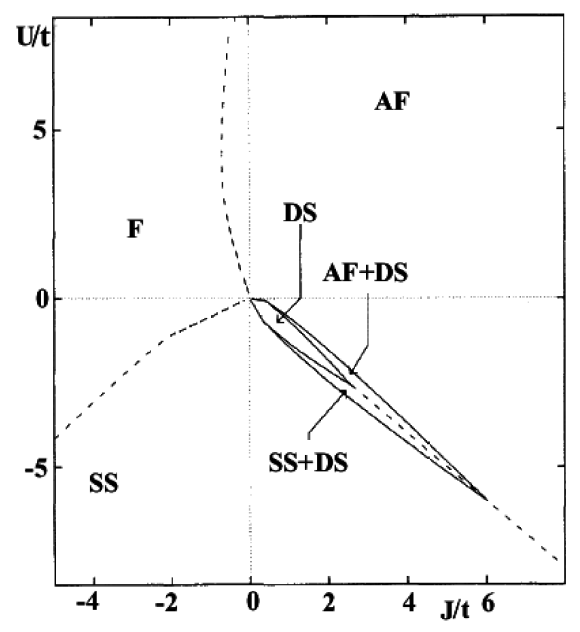

Fig. 3. Ground state diagram of the $t-U-J$ model $\left(J=J_{\perp}=J_{\|}\right)$at half-filling for $d=2 \mathrm{SQ}$ lattice calculated within (broken symmetry) HFA. Second-order phase boundaries are drawn as full lines, while first-order phase boundaries are drawn as dashed lines.

Using the results of the present research and the previous works [2-8] let us make a short summary of the possible effects of the spin-exchange interactions $J_{\perp}, J_{\|}$(Eq. (1)) and the charge exchange $I$ (Eq. (2)) in the half-filled band case. 
(1) The longitudinal spin exchange $\left(J_{\|}\right)$can stabilize uniaxial ferro (antiferro) magnetic orderings $x_{\mathrm{F}_{Z}} \neq 0$ (for $J_{\|}<0$ ), $x_{\mathrm{AF}_{\mathrm{Z}}} \neq 0$ (for $J_{\|}>0$ ), as well as, in the weak coupling limit and $J_{\|}<0$, the $\mathrm{bAF}_{X Y}$ for $d=1$ lattices, and the spin-flux (spin nematic) order $\left(\mathrm{SF}_{X Y}\right)$ for $d=2$ lattice (cf. Figs. 1, 2).

(2) The $X Y$ spin exchange $\left(J_{\perp}\right)$ favors planar ferro (antiferro) magnetic orderings with $x_{\mathrm{F}_{X Y}} \neq 0$ (for $J_{\perp}<0$ ) and $x_{\mathrm{AF}_{X Y}} \neq 0$ (for $J_{\perp}>0$ ), as well as, in the weak coupling regime and $J_{\perp}<0$, the triplet superconductivity (TS) or the $\mathrm{bAF}_{Z}$ order for $d=1$ lattices, and the charge flux (orbital AF) order, for $d=2$ lattices $[4,9]$.

(3) The attractive charge exchange $(I>0)$ can stabilize (for any dimension) the on-site s-wave pairing (SS) with $x_{S}=\frac{1}{N} \sum_{i}\left\langle c_{i \uparrow} c_{i \downarrow}\right\rangle \neq 0$, whereas the repulsive one $(I<0)$ : the eta-pairing $(\eta \mathrm{S})$, with $x_{\eta}=\frac{1}{N} \sum_{i} \exp \left(\mathrm{i} \boldsymbol{Q} \boldsymbol{R}_{i}\right)\left\langle c_{i \uparrow} c_{i \downarrow}\right\rangle \neq 0$, as well as, in the weak coupling regime, the bAF, for $d=1$ lattices, or the spin flux (spin nematic) order $\left(\mathrm{SF}_{X Y}\right)$, for $d=2$ lattices [6-9].

(4) The interplay between the on-site interaction $U$ and the intersite exchange interactions can stabilize several new ordered phases, absent at half-filling in the usual Hubbard model and in the models with $U=0$. For the models considered these phases are the following:

(i) the $t-U-I$ model in $d=1$ : the bond located CDW (bCDW) as well as three mixed phases: with site- and bond-located $\mathrm{AF}(\mathrm{sAF}+\mathrm{bAF})$, with site located $\mathrm{CDW}$ and bAF (sCDW+bAF) and with sAF+bCDW (cf. Fig. 1a in Ref. [6]),

(ii) the $t-U-J_{\|}$model in $d=1$ : the $\mathrm{AF}_{X Y}$, the bCDW and the mixed phases: $\mathrm{AF}_{X Y}+\mathrm{bAF}_{X Y}, \mathrm{bAF}_{X Y}+\mathrm{SS} / \mathrm{CDW}, \mathrm{bCDW}+\mathrm{SS} / \mathrm{CDW}$ (Fig. 2 and [9]).

(iii) the $t-U-J_{\perp}$ model in $d=1$ : the bCDW, the uniaxial antiferromagnetic $\left(\mathrm{AF}_{Z}\right)$ as well as the mixed phases: with bCDW and $s$-wave superconductivity (bCDW+SS), with triplet and singlet superconductivity (TS+SS) and with $\mathrm{TS}+\mathrm{AF}_{Z}[4,9]$.

(iv) the $t-U-J$ model $\left(J=J_{\perp}=J_{\|}\right)$in $d=2$ : the $d$-wave superconducting phase (DS) and the mixed phases AF+DS and SS+DS (cf. Fig. 3 and [5]).

The results presented have been obtained for the models at half-filling in the case of $d$-dimensional hypercubic lattices and the hopping as well as the interaction restricted to nn. As we have shown even in this simplest case the effective intersite exchange couplings can generate numerous different types of electron orderings, particularly for low dimensional lattices $\mathrm{d} \leq 2$. Important extension of the present study would be to take into account the effects of nnn hopping $t_{2}$, breaking the electron-hole symmetry of the systems considered.

\section{Acknowledgments}

We thank R. Micnas and B. Bułka for helpful discussion. This work was supported in part by the State Committee for Scientific Research, grant No. 1 P03B 084 26, 2004-2006, and by the Foundation for Polish Science. 


\section{References}

[1] R. Micnas, J. Ranninger, S. Robaszkiewicz, Rev. Mod. Phys. 62, 113 (1990).

[2] G.I. Japaridze, E. Muller-Hartmann, Phys. Rev. B 61, 9019 (2000).

[3] C. Dzurzik, G.I. Japaridze, A. Schadschneider, J. Zittartz, Eur. Phys. J. B 37, 453 (2004).

[4] W.R. Czart, S. Robaszkiewicz, Phys. Status Solidi B 243, 151 (2006).

[5] A.B. Eriksson, T. Einarsson, S. Ostlund, Phys. Rev. B 52, 3662 (1995).

[6] S. Robaszkiewicz, B. Bułka, Phys. Rev. B 59, 6430 (1999).

[7] W.R. Czart, S. Robaszkiewicz, Phys. Rev. B 64, 104511 (2001); Acta Phys. Pol. A 106, 709 (2004).

[8] S. Robaszkiewicz, W.R. Czart, Acta Phys. Pol. B 32, 3267 (2001); Phys. Status Solidi B 236, 416 (2003).

[9] W.R. Czart, S. Robaszkiewicz, in preparation.

[10] G.I. Japaridze, A.P. Kampf, M. Sekania, P. Kakashvili, Ph. Brune, Phys. Rev. B 65, 14518 (2001).

[11] M. Nakamura, Phys. Rev. B 61, 16377 (2000); J. Phys. Soc. Jpn. 68, 3123 (1999). 